\title{
Experimental Study From Chinese-Japanese And Japanese-Chinese Bilingual Behavioral Data To Brain Computer Interface
}

\author{
Xiujun $\mathrm{Li}^{1, \mathrm{a}^{*}}$, Jingjing Yang ${ }^{1, \mathrm{~b}}$ and Dan Tong ${ }^{2, \mathrm{c}}$ \\ ${ }^{1}$ The School of Computer Science and Technology, Changchun University of Science and \\ Technology, 7089 Weixing Road, Changchun, Jilin, China \\ 2Department of Radiology, No.1 Hospital of Jilin University, Jiefang Road, Changchun, Jilin, China \\ alixiujun7897@qq.com, bseiseiyou@gmail.com, ctongdan2012@126.com, "corresponding author
}

Keywords: second language; Japanese; Chinese; bilinguals

\begin{abstract}
While previous studies have compared performance between alphabetic and Chinese subjects, few data were about Japanese-speaking individuals. In this study, we used Japanese-Chinese bilingual and Chinese-Japanese bilingual subject to investigate different in processing semantic and phonological by visual judgment tasks in 15 Japanese-speaking individuals and 15 Chinese-Japanese bilingual subjects. Different patterns were observed between Japanese-speaking individuals and Chinese-Japanese bilingual subjects and used in brain computer interface (BCI).
\end{abstract}

\section{Introduction}

Recent neuroimaging studies used behavioral experiment and functional magnetic resonance imaging (fMRI) to clarify the different brain activities when bilingual speaker comprehend their first language (L1) compare with their second language(L2) [1, 2]. Many studies showed the different activation patterns of alphabetic language (e.g. English) and logographic language (e.g. Chinese characters) $[3,4,5,6]$. In this study, we used Japanese-Chinese bilingual and Chinese-Japanese bilingual subject to investigate different in processing semantic and phonological by visual judgment tasks in 15 Japanese-speaking individuals and 15 Chinese-Japanese bilingual subjects.

While previous studies have reported that Chinese-English bilinguals' alphabetic English (L2) reading is shaped by logographic Chinese (L1) and different from native English speakers'. By a study of Chinese-English bilinguals' brain activity processing of logographic Chinese (L1) and alphabetic English (L2), it is reported that reading involves language-specific neuro-cognitive systems in which alphabetic English (L2) reading is shaped by logographic Chinese (L1) for bilinguals and different from native English speakers' brain activity [1].

In this study, we used behavioral method to investigate different in processing semantic and phonological by visual judgment tasks.

\section{Methods}

Subjects. In this study, there are 15 Japanese-Chinese bilingual subjects (seven females, eight males) and 15 Chinese-Japanese bilingual (seven females, eight males). They were selected and participated in the behavioral study. We explained the details of the information form and the consent form for them before we obtained their fingerprints or signing. All subjects had normal or corrected-to normal vision. No subjects were using drugs or alcohol or had any history of neurological disease, systemic disease known to affect central nervous system functioning, clinically significant head injury or mental retardation.

Experiment Stimuli. As shown in Fig.1, forty-eight pairs of synonymous Japanese words (or Chinese words) were used for synonym judgments (Fig.1). The other 48 pairs of Japanese words (or Chinese words) were used for front size judgments. To minimize the visual similarity of stimuli, the 
two characters in each pair shared as few letters as possible. Words were commonly used in terms of their frequency.

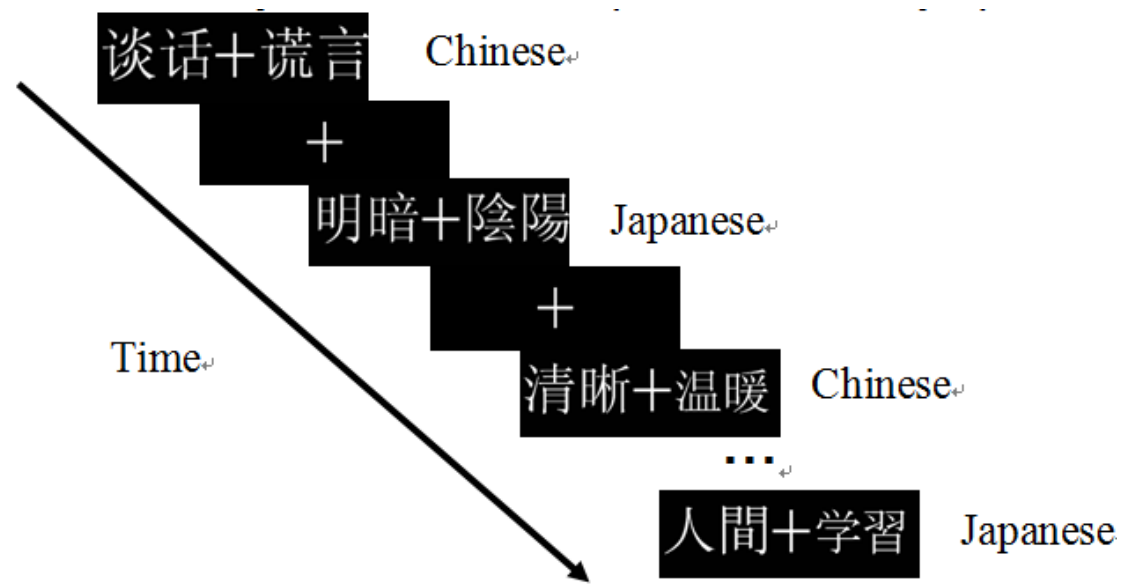

Fig. 1 In each trial, a pair of characters was exposed synchronously for $2.5 \mathrm{~s}$, one left and one right a fixation crosshair. After the presentation of the two items, a fixation crosshair was exposed for $1.5 \mathrm{~s}$. Participants indicated a positive response by pressing the key corresponding to the index finger of their right hand and a negative response by pressing the key corresponding to the index finger of their left hand.

Task and Design.The experiment stimuli were shown to subject through a display. In the phonological decision or semantic decision task, subjects decided whether the two viewed items phonologically or semantically with each other. In the font size decision task, subjects decided whether the two viewed items had a same physical size. In each trial, a pair of characters was exposed synchronously for $2,500 \mathrm{msec}$, one above and one below a fixation crosshair. After the presentation of the two items, a fixation crosshair was exposed for 1,500 msec. Bilinguals indicated a positive response by pressing the key corresponding to the index finger of their left hand and a negative response by pressing the key corresponding to the index finger of their right hand.

Data Analysis. The data were used in a repeated measures analysis of variance (ANOVA) (SPSS 16.0 for Windows) with an equal variances assumption. A Bonferroni correction at $\mathrm{P}<0.05$ was used for the post hoc multiple comparison to test the pairwise comparisons.

\section{Results}

As shown in Fig.2 and Fig.3, we calculated the mean reaction time and accuracy for each task. The mean accuracies were $97.1 \pm 1.8 \%$ for the Chinese-Japanese bilinguals with the Chinese stimuli, 92.3 $\pm 1.9 \%$ for the Chinese-Japanese bilinguals with the Japanese stimuli and $90.1 \pm 0.6 \%$ for the Japanese-Chinese bilinguals with the Japanese stimuli of semantic task; $98.1 \pm 1.9 \%$ for the Chinese-Japanese bilinguals with the Chinese stimuli,88.3 $\pm 1.9 \%$ for the Chinese-Japanese bilinguals with the Japanese stimuli and $94.1 \pm 0.6 \%$ for the Japanese-Chinese bilinguals with the Japanese stimuli of phonological task; $99.1 \pm 1.8 \%$ for the Chinese-Japanese bilinguals with the Chinese stimuli, $98.3 \pm 1.9 \%$ for the Chinese-Japanese bilinguals with the Japanese stimuli and 96.8 $\pm 0.5 \%$ for the Japanese-Chinese bilinguals with the Japanese stimuli of font size task.

The same repeated measures ANOVA and multiple comparisons were also performed for reaction time. The mean reaction time were 1480.2 \pm 1.9 for the Chinese-Japanese bilinguals with the Chinese stimuli, $1590.3 \pm 1.9$ for the Chinese-Japanese bilinguals with the Japanese stimuli and $1825.1 \pm 0.6$ for the Japanese-Chinese bilinguals with the Japanese stimuli of semantic task;1509.2 \pm 1.9 for the Chinese-Japanese bilinguals with the Chinese stimuli,1628.3 \pm 1.9 for the Chinese-Japanese bilinguals with the Japanese stimuli and $1950.1 \pm 0.6$ for the Japanese-Chinese bilinguals with the Japanese stimuli of phonological task; $825.3 \pm 1.8$ for the Chinese-Japanese bilinguals with the Chinese stimuli, $803.3 \pm 1.9$ for the Chinese-Japanese bilinguals with the Japanese stimuli and 1356.8 \pm 0.5 for the Japanese-Chinese bilinguals with the Japanese stimuli of font size task. 


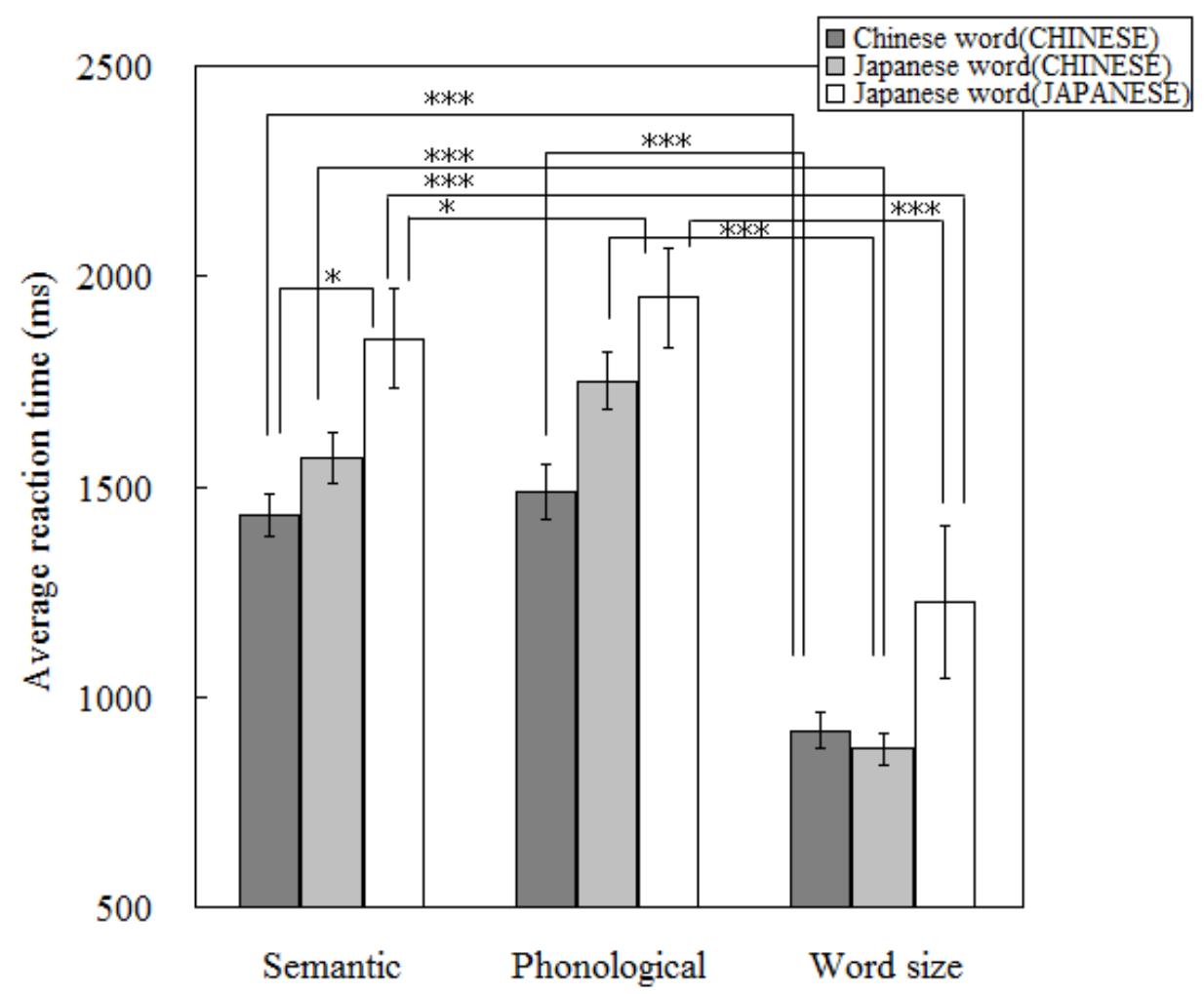

Fig. 2 average reaction time

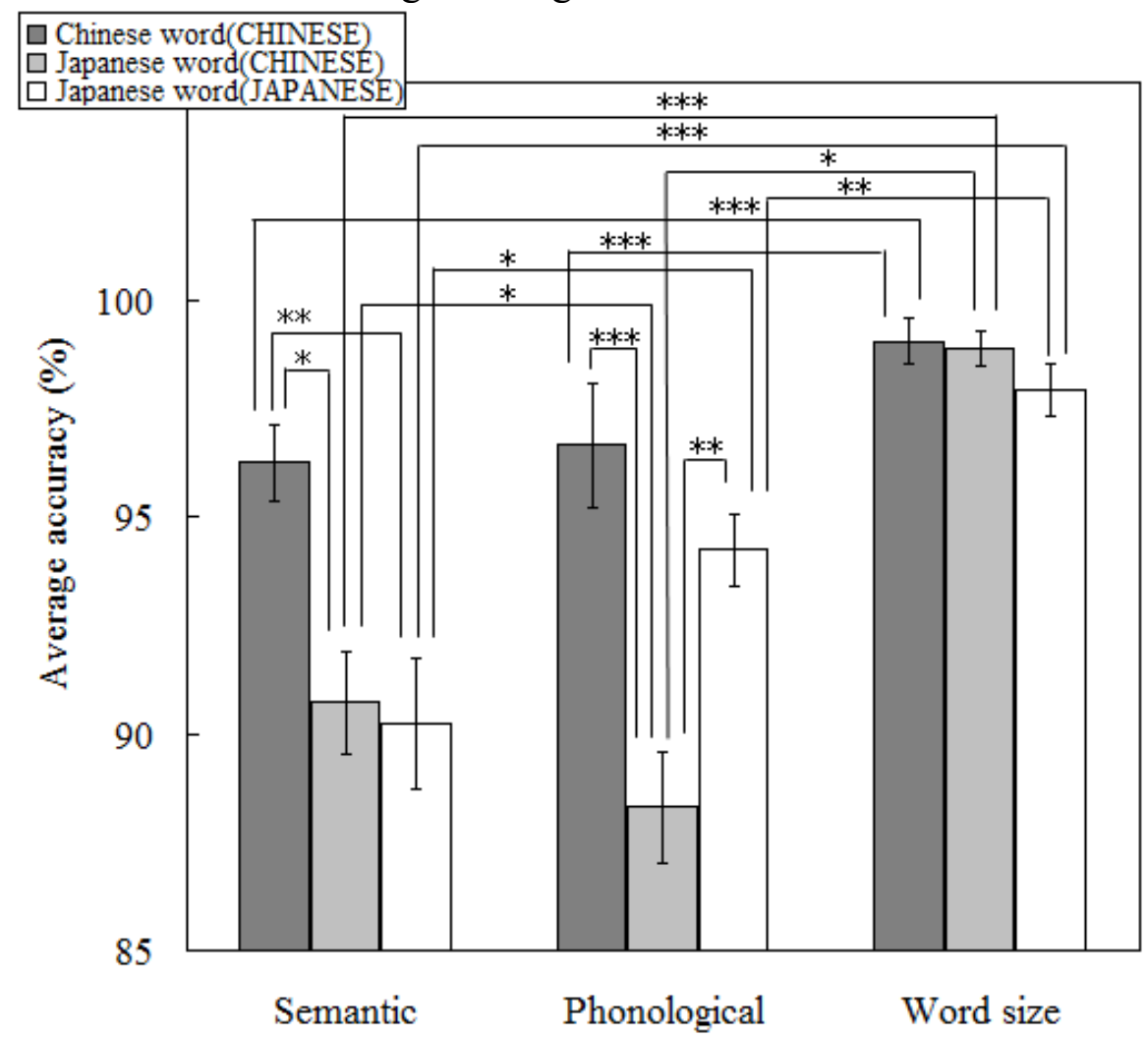

Fig. 3 average accuracy 


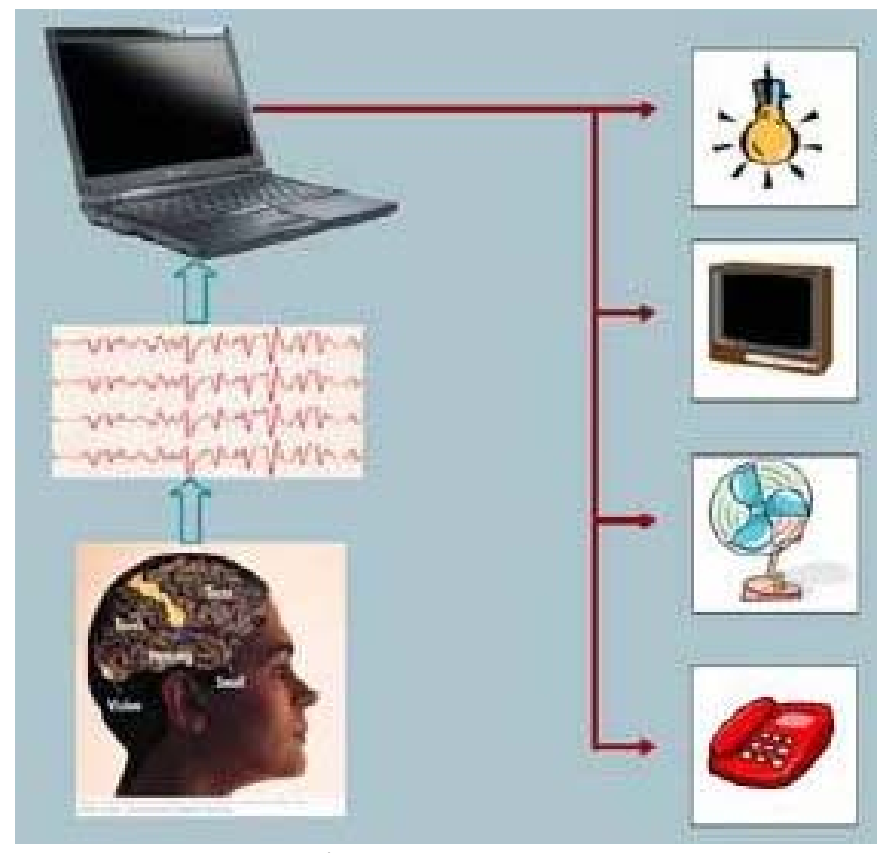

Fig.4 BCI system

\section{Discussion}

The purpose of the current second language experiment was to confirm the influence which the process of L1 affects the one of L2 between similar shape characters and for semantic and phonological processing. In this point, we found that many different were activated between L1 and L2 in our semantic or phonological tasks.

Recent neuroimging study reported that equally important is our discovery of the high similarity of neural networks involved in phonological analysis of L1 and L2 words [1]. One might expect that, as English words are linearly constructed and follow the letter-to-sound conversion rules that require elaborated phonemic processing, the left mid-superior temporal gyrus would be active. Indeed, our second fMRI experiment with native English users did find the peak activity of left inferior frontal and superior temporal gyri in phonological processing. Our failure to show activation in the mid-superior temporal gyrus when Chinese - English bilinguals processed English words phonologically is thus not due to inadequate power to detect the activation. Rather, it suggests that the processing of L1 phonology (where logographic characters are pronounced monosyllabically) carries over to L2 processing. Clearly, our Chinese subjects were applying the strategy of processing Chinese to processing English words. They did not automatically use the letter-to-sound conversion rules to pronounce English words. Collectively, our current fMRI experiments have produced the most compelling data in support of the hypothesis that language experience tunes the cortex $[5,6]$.

The present study demonstrated that different brain activation of phonological processing between Chinese-Japanese bilingual and Japanese native speakers. Future, if same Chinese tasks are applied to Chinese native speakers, we may describe about second language processing between Chinese and Japanese in greater detail, this topic is my further plan.

\section{Summary}

The behavioral result showed that our bilingual Japanese subjects have large overlaps in the neural substrates for semantic and phonological processing of Chinese (L2) and Japanese (L1) language. Different patterns were observed between Japanese-speaking individuals and Chinese-Japanese bilingual subjects. 


\section{Acknowledgement}

At last, the authors thank the people who participated in this study and the staff of Shengyang of Medical College Shengjing Hospital for their assistance with data collection. Afterwards, we gave them some science research expense. A part of this study was financially supported by (KYC-JC-XM-2015-094) and international cooperation project of Jilin provincial science and technology department(KYC-JC-XM-2016-069).

\section{References}

[1] Tan LH, John A. Spinks, Ching-Mei Feng, Wai Ting Siok, Charles A. Perfetti, Jinhu Xiong, Peter T. Fox, and Jia-Hong Gao. (2003): Neural systems of second language reading are shaped by native language. Hum Brain Mapp 18:158-166.

[2] Ulrike Halsband, (2006): Bilingual and multilingual language processing. Journal of Physiology - Paris 99 (2006) 355-369.

[3] Perani, D., Paulesu, E., Galles, N.S., Dupoux, E., Dehaene, S., Bettinardi, V., Cappa, S.F., Fazio, F., Mehler, J. (1998): The bilingual brain. Presidency age of acquisition of the second language. Brain 121 (Pt 10), 1841-1852.

[4] Satoru yokoyama,Jungho Kim,Shin-ya Uchida,Tadao Miyamoto,Kei Yoshimoto,Noriaki Yusa,Ryuta Kawashima. Left middle temporal deactivation caused by insufficient second language word comprehension by Chinese-Japanese bilinguals. Journal of Neurolinguistics 2009; 22: 467-485.

[5] Fiez JA (2000): Sound and meaning: how native language affects reading strategies. Nature Neurosci 3:3 - 5.

[6] Neville HJ, Bavelier D, Corina D, Rauschecker J, Karni A, Lalwani A, Braun A, Clark V, Jezzard P, Turner R (1998): Cerebral organization for language in deaf and hearing subjects: biological constraints and effects of experience. Proc Natl Acad Sci USA 95:922-929. 